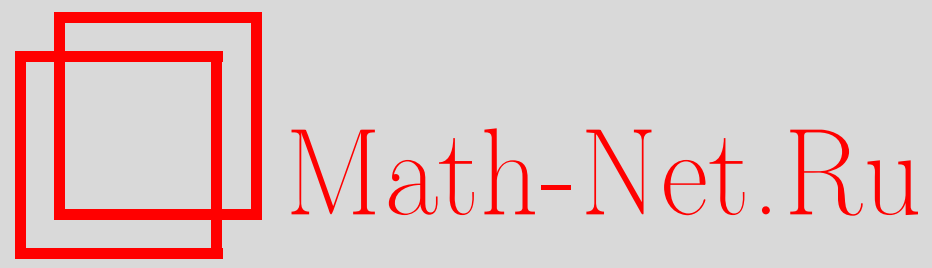

В. П. Кондаков, Существование базисов в ядерных дополняемых подпространствах пространств степенных рядов бесконечного типа, Функи. анализ и его прил., 2000, том 34, выпуск 2, 81-83

DOI: https://doi.org/10.4213/faa302

Использование Общероссийского математического портала MathNet.Ru подразумевает, что вы прочитали и согласны с пользовательским соглашением

http://www . mathnet.ru/rus/agreement

Параметры загрузки:

IP : 54.89 .56 .158

26 апреля 2023 г., 14:21:34

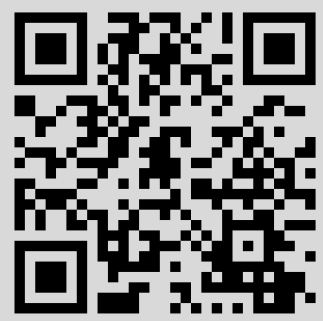


В завершение отметим, что условие (4) для оператора (5) сводится к проверке ограниченности некоторых сингулярных интегральных операторов в пространстве $L_{2}(\mathbb{R})$. А именно, справедлива

ТЕОРема 3. Если спектр одномерного возмущения оператора умножения на независимую переменную (5) абсолютно непрерьвен $u(\varphi, \psi)=0$, то для подобия этого оператора самосопряженному оператору достаточно, чтобы сингулярнье интегральнье операторь с ядрами

$$
\begin{aligned}
& T_{1}(k, t)=\frac{i \overline{\psi(t)}}{t-k-i 0}+\frac{1-i\|\varphi\|\|\psi\|^{-1}\left(\psi(x)(x-k-i 0)^{-1}, \psi(x)\right)}{D(k+i 0)} \frac{\overline{\varphi(t)}}{t-k-i 0}, \\
& T_{2}(k, t)=\frac{i \overline{\varphi(t)}}{t-k-i 0}+\frac{1-i\|\psi\|\|\varphi\|^{-1}\left(\varphi(x)(x-k-i 0)^{-1}, \varphi(x)\right)}{D_{*}(k+i 0)} \frac{\overline{\psi(t)}}{t-k-i 0},
\end{aligned}
$$

где $D_{*}(\lambda) \equiv \overline{\overline{D(\bar{\lambda})}}$, бьли ограниченьл в пространстве $L_{2}(\mathbb{R})$.

Авторы выражают благодарность С. Н. Набоко за внимание к работе.

\title{
ЛИТЕРАТУРА
}

1. Набоко C. Н. Функц. анализ и его прил., 18, вып. 1, 16-27 (1984). 2. Van Casteren J. Pacif. J. Math., 104, No. 1, 241-255 (1983). 3. Фаддеев M. М. Функц. анализ и его прил., 26, вып. 4, 80-83 (1992). 4. Секефальви-Надь Б., Фояш Ч. Гармонический анализ операторов в гильбертовом пространстве. Мир, М., 1970. 5. Павлов Б. С. Изв. АН СССР, сер. матем., 39, 123-148 (1975). 6. Набоко С. Н. Труды ММО, 147, 86-114 (1980). 7. Бродский M. C. Треугольные и жордановы представления линейных операторов. Наука, М., 1969. 8. Сахнович Л. А. Изв. АН СССР, сер. матем., 33, № 1, 52-64 (1969). 9. Веселов В. Ф. Вестник ЛГУ, сер. 1, вып. 4, $19-24$ (1988). 10. Веселов В. Ф. Вестник ЛГУ, сер. 1, вып. 2, 11-17 (1988).

Санкт-Петербургский государственный университет, физический факультет

Поступило в редакцию 14 августа 1998 г.

УДК 513.881

\section{Существование базисов в ядерных дополняемых подпространствах пространств степенных рядов бесконечного типа*}

\author{
(c) 2000. В. П. КондАков
}

Проблема существования базиса в произвольном дополняемом подпространстве ядерного пространства степенных рядов бесконечного типа, поставленная Б. С. Митягиным, решалась в работах [1-3] только при различных добавочных ограничениях на пространства или на их дополняемые подпространства.

Конкретными и широко используемыми в исследованиях пространствами степенных рядов бесконечного типа являются пространство быстро убывающих функций или его реализация в виде пространства $s$ быстро убывающих

*Работа выполнена при частичной финансовой поддержке фонда РФФИ, грант № 97-0100215. 
последовательностей, пространства целых функций с топологией равномерной сходимости на компактах и другие.

Пространством степенных рядов бесконечного типа называют пространство Кёте числовых последовательностей

$$
l_{p}\left[\exp r b_{n}\right]=\left\{\xi=\left(\xi_{n}\right):\left(\sum_{n}\left|\xi_{n}\right|^{p} \exp r p b_{n}\right)^{1 / p}=|\xi|_{r}<\infty, r \in \mathbb{N}\right\},
$$

$1 \leqslant p \leqslant \infty$, топология которого задана системой норм $\left(|\cdot|_{r}\right)$.

Условие ядерности пространства $l_{p}\left[\exp r b_{n}\right]$ состоит в том, что для некоторого числа $A>0$

$$
\sum_{n} \exp \left(-A b_{n}\right)<+\infty .
$$

При изучении базисов и структурных вопросов для пространств степенных рядов бесконечного типа были найдены линейные топологические инварианты - геометрические свойства $D_{1}$ и $\Omega$, присущие всем пространствам степенных рядов бесконечного типа и их дополняемым подпространствам [4-6].

Для случая ядерного пространства степенных рядов бесконечного типа $E$, изоморфного своему декартову квадрату, известны необходимые и достаточные условия в терминах асимптотик $n$-поперечников окрестностей нуля (диаметральной размерности), при которых пространство Фреше со свойствами $D_{1}$ и $\Omega$ изоморфно дополняемому подпространству в $E$ (см. [6]).

Приведем ниже общий результат, из которого вытекает полное решение проблемы существования базиса в произвольном дополняемом подпространстве пространства $s=l_{2}\left[n^{r}\right]$ быстро убывающих последовательностей, равно как и полное решение проблемы описания дополняемых подпространств ядерных пространств степенных рядов бесконечного типа. Помимо прочего, в упомянутом результате из [6] снимается требование изоморфизма пространства $E$ своему декартову квадрату.

Напомним определения геометрических свойств $D_{1}$ и $\Omega$.

ОПРедЕЛЕниЕ $1[4,5]$. Пространство Фреше $\left(E,\left(|\cdot|_{r}\right)\right)$ обладает свойством $D_{1}$ (в другой терминологии $D N$ ), когда выполнено следующее условие: существует непрерывная норма $\|\cdot\|$, такая, что для каждого $r \in \mathbb{N}$ найдутся $s(r) \in \mathbb{N}$ и $c(r)>0$, при которых

$$
|\cdot|_{r}^{2} \leqslant c(r)\|\cdot\||\cdot| s(r) \cdot
$$

Пусть $\left\{U_{r}\right\}$ - базис замкнутых абсолютно выпуклых окрестностей нуля в пространстве $\Phi$ реше $E$, т. е. $U_{r}=\left\{e \in E:|e|_{r} \leqslant 1\right\}, r \in \mathbb{N}$.

ОПредЕЛЕниЕ $2[6]$. Пространство Фреше $E$ обладает свойством $\Omega$, когда для каждого $r \in \mathbb{N}$ найдется такое $s(r) \in \mathbb{N}$, что

$$
U_{s(r)} \subset C t^{m} U_{k}+\frac{1}{t} U_{r}, \quad t>0
$$

для любого $k \in \mathbb{N}$ и некоторых $m, C>0$.

Основной результат формулируется в следующей теореме.

Теорема 1. Пусть ядерное пространство Фреше Е обладает свойствами $D_{1}$ и $\Omega$. Тогда оно имеет базис и изоморфно пространству степенньх рядов бесконечного типа. 
Поскольку любое дополняемое подпространство пространства степенных рядов бесконечного типа обладает свойствами $D_{1}$ и $\Omega$, из теоремы 1 и результатов статьи [7] выводится следующий факт.

Теорема 2. В пространстве степенных рядов бесконечного типа каждое ядерное дополняемое подпространство имеет базис и изоморфно некоторому координатному подпространству.

Из теоремы 2 непосредственно следует положительное решение проблемы существования базиса в произвольном дополняемом подпространстве пространства $s$ быстро убывающих последовательностей.

Из теоремы 1 и результатов статьи [7] легко также вывести, что ядерное пространство Фреше $E$, обладающее свойствами $D_{1}$ и $\Omega$, изоморфно заданному пространству $F=l_{2}\left[\exp r b_{n}\right]$ тогда и только тогда, когда линейные размерности пространств $E$ и $F$ совпадают или равны их диаметральные размерности (определение см., например, в $[4,6,7]$ ). Аналогичным образом можно сформулировать и условие существования изоморфизма ядерного пространства Фреше со свойствами $D_{1}$ и $\Omega$ на дополняемое подпространство заданного пространства степенных рядов бесконечного типа в терминах определяющих эти пространства матриц Кёте.

\section{ЛИТЕРАТУРА}

1. Dubinsky E., Vogt D. Studia Math., 93, No. 1, 71-85 (1989). 2. Aytuna A., Krone J., Terzioglu T. Math. Ann., 283, 193-202 (1989). 3. Krone J. Proc. Amer. Math. Soc., 105, No. 2, 350-355 (1989). 4. Драгилев М. М. Матем. сб., 58, № 2, 153-173 (1965). 5. Zahariuta V. P. Studia Math., 46, 201-221 (1973). 6. Vogt D., Wagner M. J. Studia Math., 70, 63-80. 7. Кондаков В. П. Studia Math., 76, 137-151 (1983).

Ростовский государственный университет

Поступило в редакцию 18 мая 1998 г.

УДК 512.2

\section{Функторы и диадические множества конечного типа}

(C) 2000. Л. А. Назарова, А. В. РойтеР

К 85-летию И. М. Гельфанда

Пусть $\Phi: \mathscr{A}^{\circ} \times \mathscr{B} \rightarrow \mathscr{C}-$ аддитивный функтор и $\mathscr{C} \subset \operatorname{Mod} k$, где $k-$ кольцо. Построим категорию $\Phi^{\triangle}$ следующим образом: $\mathrm{Ob}^{\triangle}=\{(A, B, \varphi) \mid$ $A \in \mathscr{A}, B \in \mathscr{B}, \varphi \in \Phi(A, B)\}, \Phi^{\triangle}\left(\left(A_{1}, B_{1}, \varphi_{1}\right),\left(A_{2}, B_{2}, \varphi_{2}\right)\right)=\{(\alpha, \beta) \mid \alpha \in$ $\left.\mathscr{A}\left(A_{1}, A_{2}\right), \beta \in \mathscr{B}\left(B_{1}, B_{2}\right), \varphi_{1} \Phi\left(1_{A_{1}}, \beta\right)=\Phi\left(\alpha, 1_{B_{2}}\right) \varphi_{2}\right\}$ (см. [1]). Если $\mathscr{A}=\mathscr{B}=\mathscr{C}$ и $\Phi=\operatorname{Hom}_{\mathscr{C}}$, то $\Phi^{\triangle}$ есть категория $\mathscr{C}^{\triangle_{1}}$ морфизмов категории $\mathscr{C}$ (см. [2]). Если $F: \mathscr{B} \rightarrow \mathscr{C}$ - аддитивный функтор (от одной переменной), то построим $\Phi_{F}: \mathscr{C}^{\circ} \times \mathscr{B} \rightarrow \operatorname{Mod} k, \Phi_{F}(C, B)=\operatorname{Hom}_{\mathscr{C}}(C, F(B))$, и положим $F^{\nabla}=\left(\Phi_{F}\right)^{\triangle}$. В дальнейшем $\mathscr{C}$ - это категория $\bmod k$ конечно порожденных $k$-модулей.

Существенность этих категорий очевидна. В частности, к $F^{\nabla}$ сводится описание представлений конечномерных алгебр [3]. 